\title{
Filtros digitales de tangente hiperbólica aplicados al alisado de las perturbaciones en los datos de resistividad de fosfato de Marruecos
}

\author{
S. Bakkali ${ }^{*}$ y M. Amrani ${ }^{2}$ \\ ${ }^{1}$ Geosciences \& Environment Group, Abdelmalek Essaadi University \\ PO Box 416, Tangier, Morocco \\ E-mail: saad.bakkali@menara.ma \\ ${ }^{2}$ Engineering Process Department, Abdelmalek Essaadi University \\ PO Box 416, Tangier, Morocco \\ E-mail: amrani.mahacine@menara.ma
}

(recibido/received: 10-Dic-2007; aceptado/accepted: 30-Abril-2008)

\begin{abstract}
RESUMEN
Es posible diseñar filtros pasa bajos y pasa bandas por medio de una combinación de funciones tangente hiperbólica en el dominio de la frecuencia, usando los teoremas de escalamiento y deslizamiento de las transformadas de Fourier. Las funciones de filtro correspondientes en el dominio del tiempo pueden ser derivadas analíticamente a partir de las expresiones en el dominio de la frecuencia. Los parámetros de suavidad controlan las pendientes en las regiones de corte y permiten la construcción de filtros relativamente pequeños al mismo tiempo que reducen las oscilaciones de la respuesta del filtro en el dominio del tiempo. Se pueden elegir diferentes parámetros de suavidad para las frecuencias de corte alta y baja en el diseño de filtros pasa banda. Siguiendo el esquema propuesto en este artículo se pueden derivar fácilmente los otros tipos de filtro.
\end{abstract}

Palabras claves: filtro digital; fosfato; hiperbólico; Marruecos; resistividad

\begin{abstract}
Low- and band-pass filters can be designed by a combination of hyperbolic tangent functions in the frequency domain using the scaling and shifting theorems of the Fourier transforms. The corresponding filter function in the time domain can be derived analytically from the frequency domain expression. The smoothness parameters control the slopes at the cut off regions and permit the construction of a relatively short filter while reducing the oscillations of the filter response in the time domain. Different smoothness parameters can be chosen for the low and high cut off frequencies in the band-pass filter design. Following the proposed scheme can easily derive the other type of the filters.
\end{abstract}

Keywords: digital filter; hyperbolic; Moroccan; phosphate; resistivity

*Autor para la correspondencia 


\section{INTRODUCCIÓN}

Actualmente varios métodos son utilizados para optimizar los bordes y contornos de los mapas de datos geofísicos. Con la ayuda de un mapa de resistividad se esperaba graficar en 2 dimensiones la señal eléctrica de resistividad, en una inspección de resistividad realizada en Marruecos en el campo de la minería. Zonas anómalas de "perturbaciones" de los depósitos de fosfato, corresponden a anomalías de la resistividad. Proponemos un nuevo método para la reducción del ruido blanco causado por las perturbaciones en el mapa de datos de resistividad de fosfato Marroquí, basado en el uso del método de filtros de funciones de tangente hiperbólica (FFTH). La efectividad de nuestro método para la reducción exitosa del ruido y el alisado de los datos de resistividad, ha sido probada con mucho éxito en el análisis de datos geofísicos constantes.

La base de datos de resistividad usada fue una recopilación de 51 transversales a un espaciamiento de $20 \mathrm{~m}$. Se colocaron 101 estaciones a $5 \mathrm{~m}$ de distancia por cada transversal, lo cuál hace un total de 5151 estaciones usadas en conjunto en la exploración de la resistividad, las cuales representaban en un mapa las zonas anómalas de fosfato $o$ "perturbaciones". La presente aplicación trata del análisis de los mapas de resistividad utilizando la técnica de alisado por medio de FFTH para remover el ruido en zonas anómalas del mapa de resistividad de los depósitos de fosfato. Los FFTH constituyen una herramienta eficiente en la interpretación de datos geofísicos, particularmente apropiada para la reducción de ruido, filtrado y análisis de singularidades en los datos de resistividad. La técnica de alisado mediante los FFTH demostró ser consistentemente útil al ser aplicada a las perturbaciones en los datos de resistividad de fosfato de Marruecos.

\section{EL CONTEXTO MATEMÁTICO}

Al combinar dos funciones tangentes hiperbólicas en el dominio de la frecuencia se puede construir una expresión analítica, la cuál proporciona los coeficientes del filtro para la evaluación numérica de las integrales tipo Hankel a como sigue (Johansen y Sørensen, 1979):

$P(v)=\frac{1}{2} \tanh \left[\frac{\pi}{a}\left(v+\frac{1}{2}\right)\right]-\frac{1}{2} \tanh \left[\frac{\pi}{a}\left(v-\frac{1}{2}\right)\right]$

donde $v$ y $a$ denotan la frecuencia y el parámetro de 'suavidad', respectivamente. El parámetro de suavidad $a$ es una pequeña constante que toma valores menores a la unidad.

La función $P(v)$ es denominada la "función-P" y es analítica en todo el espacio desde $-\infty$ a $+\infty$ y no tiene discontinuidades. La forma de la "función-P" se asemeja a la de una caja cuadrada y consecuentemente la función en el dominio del tiempo se asemeja a la función-sen $(c)$. La transformada inversa de Fourier de la ecuación (1) se puede escribir (Johansen y Sørensen, 1979),

$P(t)=\frac{a \cdot \operatorname{sen}(\pi t)}{\operatorname{senh}(\pi a t)}$

La función $P(t)$ es referida como la función$\operatorname{sen}(s h)$. El par de transformadas de Fourier antes mencionadas son utilizadas también con el mismo propósito, es decir como una función truncante en el dominio de la frecuencia y como una función interpolante en el dominio del tiempo (Christensen, 1990; Sørensen y Johansen, 1994).

El artículo presenta el uso potencial de los FFTH pasa bajos y pasa banda aplicados a la reducción de ruido y suavizado de las respuestas de los datos de resistividad de fosfatos Marroquí a las perturbaciones (Bakkali, 2005, 2006, 2007; Bakkali y Bahi, 2006).

\section{El filtro pasa bajos}

Se puede desarrollar una expresión para el filtro pasa bajos haciendo uso del siguiente par de transformadas de Fourier (Bracewell, 1965):

$\frac{i}{\operatorname{senh}(\pi t)} \leftrightarrow \tanh (\pi v)$ 
donde $i^{2}=-1$. Al aplicar la propiedad de escalamiento de la transformada de Fourier se encuentra que,

$$
h(t)=\frac{i a v_{L}}{\operatorname{senh}\left(2 \pi v_{L} t\right)} \leftrightarrow H(v)=\frac{1}{2} \tanh \left[\frac{\pi v}{2 a v_{L}}\right]
$$

donde $v_{L}$ es una constante y será utilizada como la frecuencia de corte del filtro pasa bajos en el desarrollo que sigue. La Fig. 1 nos muestra una combinación de dos funciones tangentes hiperbólicas dando la deseada expresión del filtro pasa bajos,

$$
H_{L}(v)=A\left[H\left(v+v_{L}\right)-H\left(v-v_{L}\right)\right]
$$

donde A es la ganancia del filtro. Las amplitudes de la entrada no son modificadas si A se escoge igual al intervalo de muestreo.

Sustituyendo la ecuación (4) en la ecuación (5) nos da la expresión final:

$$
H_{L}(v)=\frac{A}{2}\left[\tanh \left(\frac{\pi\left(v+v_{L}\right)}{2 a v_{L}}\right)-\tanh \left(\frac{\pi\left(v-v_{L}\right)}{2 a v_{L}}\right)\right]
$$
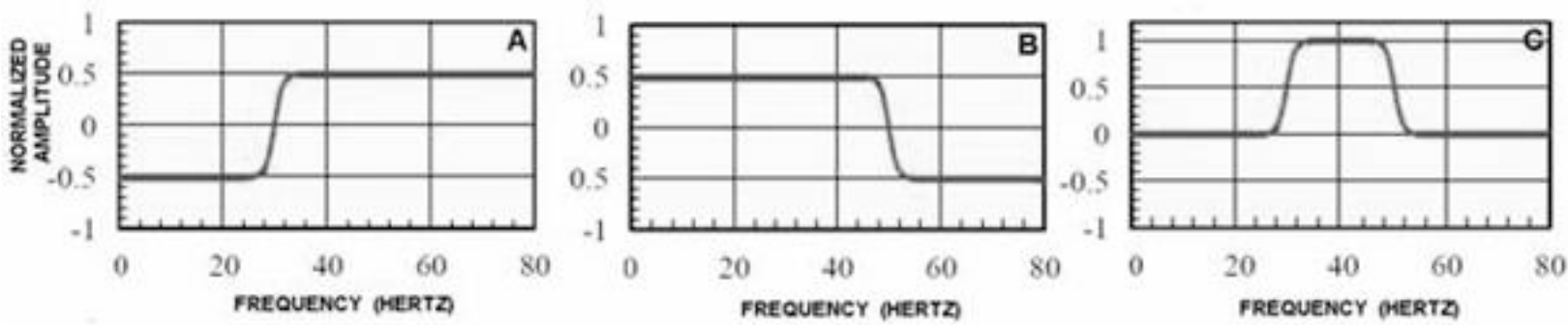

Fig. 1 Un filtro pasa bajos de tangente hiperbólica en el dominio de la frecuencia. La combinación de las funciones $H\left(v+v_{L}\right)[\mathrm{A}] \mathrm{y}-H\left(v-v_{L}\right)$ [B] derivadas a partir de la ecuación (4) proporcionan el filtro pasa bajos deseado $H(v)[\mathrm{C}]$.

Los coeficientes del filtro pueden ser calculados a partir de la equivalencia en el dominio del tiempo de la ecuación (6). Se puede derivar fácilmente una expresión para este propósito de las ecuaciones (4) y (5) utilizando el teorema de deslizamiento de la transformada de Fourier (Bracewell, 1965),

$$
h_{L}(t)=h(t)\left(e^{-2 \pi i v_{L} t}-e^{2 \pi i v_{L} t}\right)
$$

el cuál se transforma en,

$$
h_{L}(t)=2 A a v_{L} \frac{\operatorname{sen}\left(2 \pi v_{L} t\right)}{\operatorname{senh}\left(2 \pi a v_{L} t\right)}
$$

Las ecuaciones (6) y (8) sirven para el cálculo de la función de transferencia del filtro en el dominio de la frecuencia y la respuesta del filtro en el dominio del tiempo respectivamente. El parámetro de suavidad controla la pendiente de la función de transferencia del filtro alrededor de la frecuencia de corte. Si este parámetro toma valores muy pequeños, entonces $H_{L}(v)$ se asemeja a la respuesta de un filtro ideal representada por una caja cuadrada (ver Fig. 2).

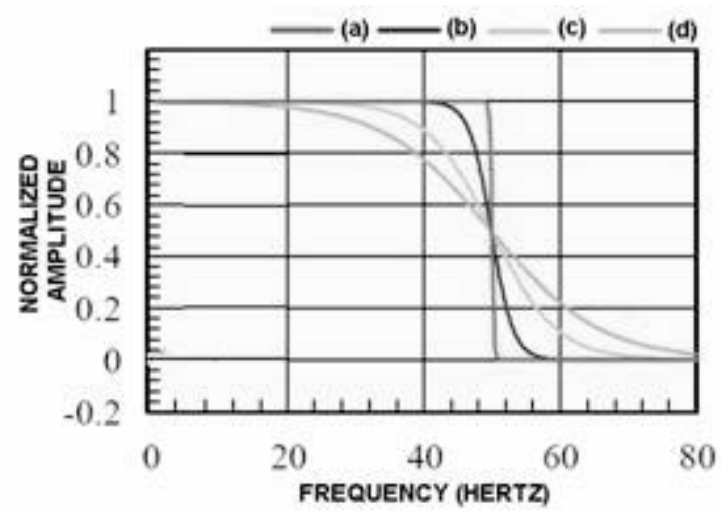

Fig. 2 Filtro FTH pasa bajos [parámetros de suavidad $(\mathrm{a})=0.01,(\mathrm{c})=0.05,(\mathrm{c})=0.3,(\mathrm{~d})=$ $0.5]$. 
Consecuentemente, la respuesta del filtro en el dominio del tiempo se aproxima a la función$\operatorname{sen}(c)$ ya que $\operatorname{senh}(x) \cong x$ para argumentos pequeños. Si el parámetro de suavidad toma valores relativamente altos, la pendiente del filtro en el dominio de la frecuencia decrece. Esto reducirá significativamente las oscilaciones de la respuesta en el tiempo del filtro y permite la construcción de un filtro relativamente pequeño en longitud (ver Fig. 3). Se requiere un cuidado especial en la selección del parámetro de suavidad porque un valor muy alto puede resultar en una inconveniente extracción de componentes de frecuencias no deseadas en la señal de entrada. Sin embargo el fácil control del grado de atenuación en la banda de transición es siempre posible.

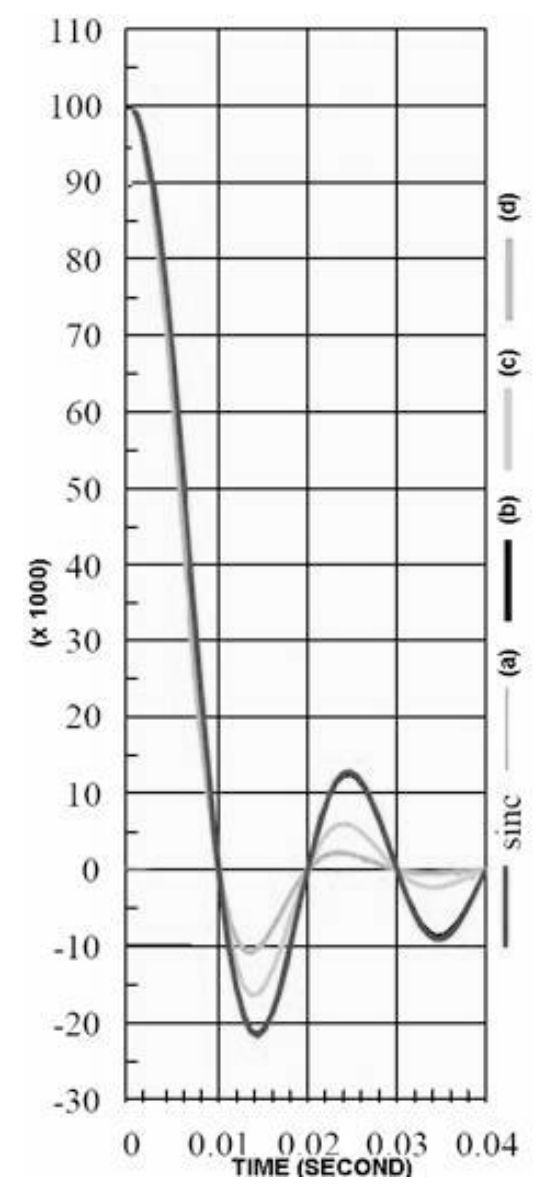

Fig. 3 Comparación de la función-sen $(c)$ y las respuestas en el tiempo de filtros FTH pasa bajos dados en la Fig. 2. Los parámetros de suavidad controlan las oscilaciones de las respuestas senh.

\section{El filtro pasa banda}

Un filtro pasa banda puede obtenerse sustrayendo un filtro pasa bajos de otro filtro que tenga una frecuencia de corte diferente. De esta manera, la función de transferencia del filtro pasa banda puede ser obtenida al combinar 4 funciones tangente hiperbólica a como se muestra en la ecuación (9),

$$
\begin{aligned}
H_{B}(v) & =\frac{A}{2}\left[\tanh \left(\frac{\pi\left(v+v_{H}\right)}{2 a_{2}\left(v_{H}-v_{L}\right)}\right)-\tanh \left(\frac{\pi\left(v-v_{H}\right)}{2 a_{2}\left(v_{H}-v_{L}\right)}\right)\right] \\
& -\frac{A}{2}\left[\tanh \left(\frac{\pi\left(v+v_{L}\right)}{2 a_{1}\left(v_{H}-v_{L}\right)}\right)+\tanh \left(\frac{\pi\left(v-v_{L}\right)}{2 a_{1}\left(v_{H}-v_{L}\right)}\right)\right]
\end{aligned}
$$

donde $v_{L}$ y $v_{H}$ son las frecuencias de corte alta y baja, $a_{1} \mathrm{y} a_{2}$ son los parámetros de suavidad. Los diferente valores numéricos de los parámetros de suavidad en $(a)$ y $(b)$ permiten el ajuste independiente de las pendientes en la banda de transición (ver Fig. 4).

La transformada inversa de Fourier de la ecuación (9) proporciona la respuesta en el dominio del tiempo,

$$
\begin{aligned}
h_{B}(t)= & 2 A\left(v_{H}-v_{L}\right)\left\{\frac{a_{2} \cdot \operatorname{sen}\left(2 \pi v_{H} t\right)}{\operatorname{senh}\left(2 \pi a_{2} t\left(v_{H}-v_{L}\right)\right)}\right. \\
& \left.-\frac{a_{1} \cdot \operatorname{sen}\left(2 \pi v_{L} t\right)}{\operatorname{senh}\left(2 \pi a_{1} t\left(v_{H}-v_{L}\right)\right)}\right\}
\end{aligned}
$$

Los valores muestreados de la ecuación (10) nos dan los coeficientes deseados del filtro (ver Fig. 5). Las otras propiedades del filtro pasa banda son las mismas de los filtros pasa bajos. Las propiedades sobresalientes del filtro sugerido pueden resumirse como sigue:

La función de transferencia de los FFTH es analítica en todo el espacio desde $-\infty$ a $+\infty$ y no posee discontinuidades. Los parámetros de suavidad controlan las pendientes en las frecuencias de corte y diferentes parámetros de suavidad pueden escogerse para las frecuencias de corte baja y alta en el diseño de filtros pasa banda. Las funciones correspondientes en el dominio del tiempo pueden ser derivadas analíticamente a partir de las expresiones en el 
dominio de la frecuencia y permiten la construcción de filtros relativamente pequeños al tiempo que reducen las oscilaciones de la respuesta del filtro en el dominio del tiempo.

El fácil control de la pendiente de la función de transferencia y la supresión de rizado de la función de respuesta son las principales ventajas de los filtros sugeridos.

La decisión concerniente acerca de los valores del parámetro de suavidad puede hacerse interactivamente dependiendo del problema en cuestión.
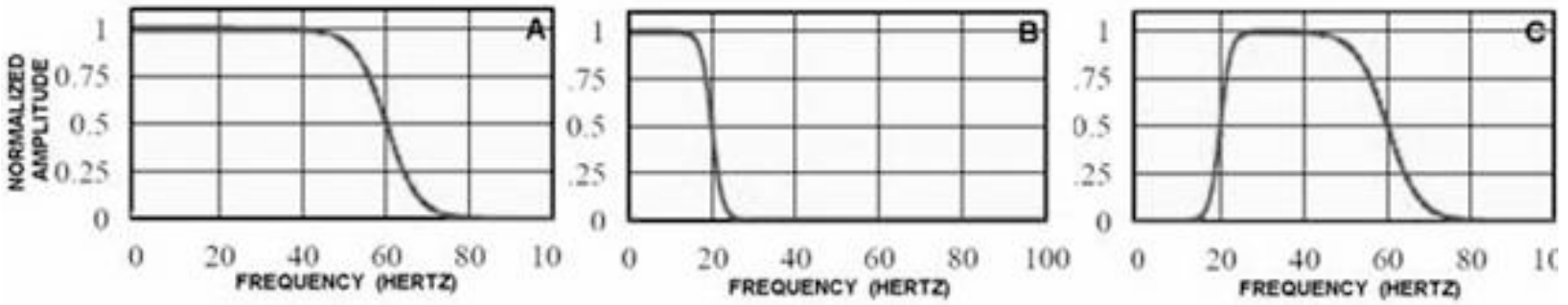

Fig. 4 Un filtro FTH pasa banda en el dominio de la frecuencia como la suma de los filtros pasa bajos mostrados en (A) y (B) poseyendo frecuencias de corte de 60 y $20 \mathrm{~Hz}$ y parámetros de suavidad de $0.3 \mathrm{y}$ 0.01 , respectivamente. El filtro pasa banda resultante tiene pendientes diferentes en las regiones de corte alta y baja.
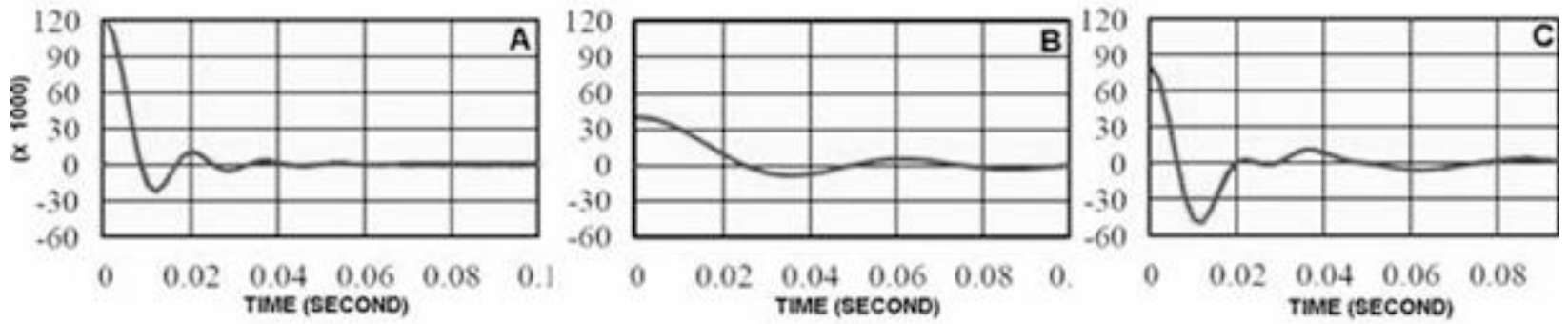

Fig. 5 Respuestas en el dominio del tiempo de un filtro pasa banda (A), (B) y (C) corresponden a las representaciones en el dominio del tiempo de las funciones de transferencia de la Fig. 4 [(A), (B), (C)].

\section{APLICACIONES}

Los datos de resistividad recolectados en la exploración son a menudo contaminados con ruido $\mathrm{y}$ artefactos provenientes de fuentes variadas. La presencia de ruido en los datos de resistividad distorsionan las características de la señal geofísica, resultando en un pobre procesamiento subsecuente.

Consecuentemente, el primer paso en cualquier procesamiento de tales datos geofísicos es la "limpieza" del ruido de forma tal que se preserven las variaciones abruptas de la señal. El alisamiento utilizando FFTH se ha convertido en una poderosa herramienta de procesamiento de imágenes y señales que ha encontrado aplicaciones en muchas áreas de la ciencia.

Este método, es una técnica usada ampliamente que es aplicable al alisado de datos geofísicos de manera más efectiva que uno de los métodos usados recientemente (Bakkali, 2007).

La presente aplicación está relacionada con el análisis de mapas de datos de resistividad, utilizando el alisamiento provisto por los filtros FTH para reducir el ruido en las zonas anómalas del mapa de la señal de resistividad de las perturbaciones de los depósitos de fosfato (ver Fig. 6). 


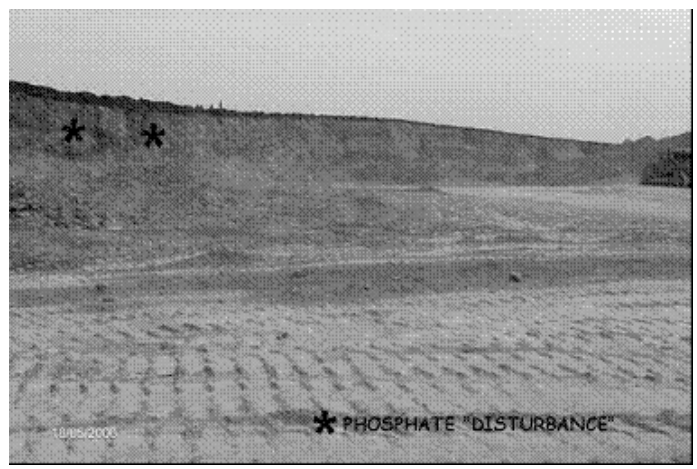

Fig. 6 Ejemplo de "perturbación" afectando los estratos de fosfato de Marruecos.

La base de datos de resistividad usada fue una recopilación de 51 transversales (ver Fig. 7) a un espaciamiento de $20 \mathrm{~m}$. Se colocaron 101 estaciones a $5 \mathrm{~m}$ de distancia por cada transversal, lo cuál hace un total de 5151 estaciones usadas en conjunto en la exploración de la resistividad, las cuales representaban a través de un mapa las zonas anómalas de fosfato o "perturbaciones" (ver Fig. 8) (Bakkali, 2005, 2006, 2007).

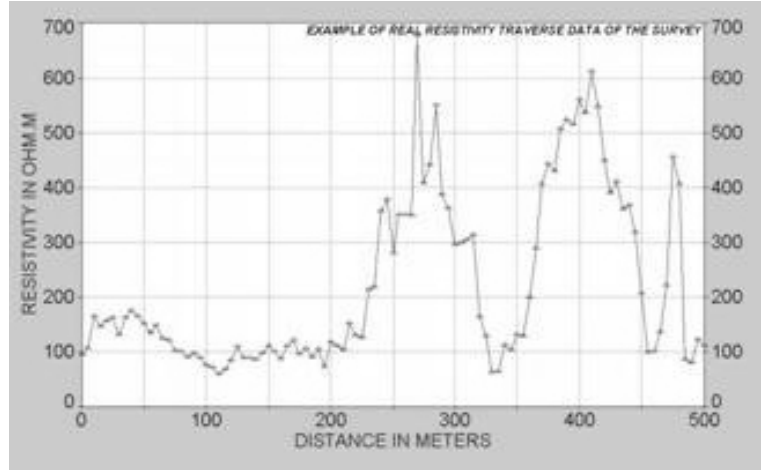

Fig. 7 Ejemplo de datos reales de resistividad transversal de la exploración.

Calculamos la salida del filtro digital FTH para cada resistividad transversal (ver Fig. 9). Posteriormente utilizamos todos los resultados para construir un mapa regular que representa de hecho un mapa de filtro FTH de las perturbaciones de depósitos de fosfato (ver Fig. 10). La ventaja de usar este filtro es la habilidad de preservar los momentos de mayor orden en los datos de resistividad y de esta manera reducir el alisamiento de la altura de los picos. En una poderosa herramienta apropiada para la remoción del ruido y alisado de los datos de resistividad. Además esta propiedad es crucial para realizar la reconstrucción de la señal geofísica filtrada correspondiente a mapa de datos de anomalías de datos de resistividad de las perturbaciones de depósitos de fosfato de Marruecos.

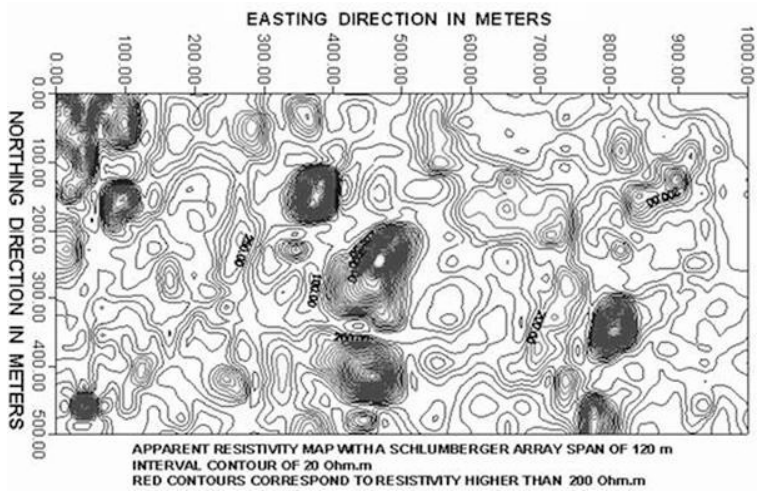

Fig. 8 Un mapa de las zonas de fosfato con perturbaciones de Marruecos.

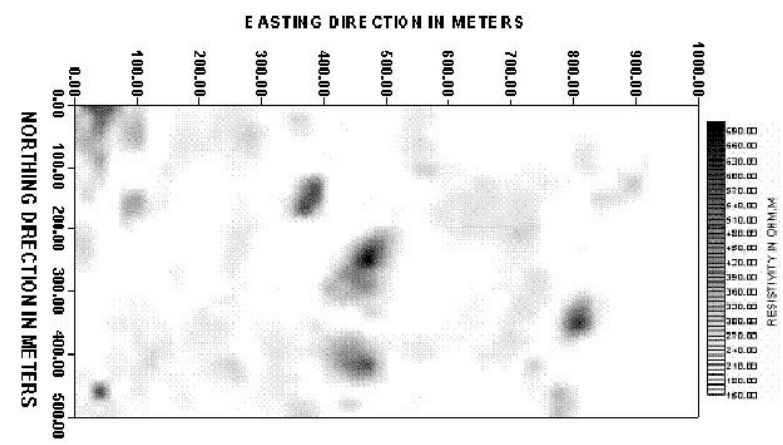

Fig. 9 Salida del filtro FTH correspondiente a los datos de resistividad.

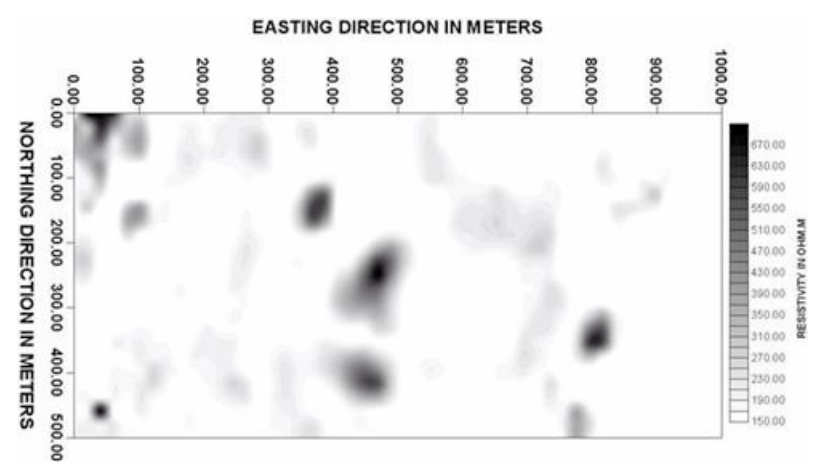

Fig. 10 La salida del filtro FTH del mapa de las perturbaciones de depósitos de fosfato dado en la Fig. 6. 


\section{CONCLUSIONES}

La Fig. 10 representa un indicador del nivel alisado en la variación de contraste de densidad entre las perturbaciones y la roca normal portadora de fosfato. El mapa de salida del filtro FTH correspondiente al modelado de las perturbaciones de resistividad fueron obtenidas mediante una rutina de AutoSignal. El uso del filtrado FTH representa un método efectivo de filtrado que hace posible atenuar considerablemente el ruido representado por las perturbaciones aleatorias menores y dispersas. Comparativamente a los métodos clásicos usados en el filtrado y reducción de ruido de los mapas de datos geofísicos, la ventaja de los filtros FTH es la de que no introducen una distorsión significativa a la forma de la señal de resistividad original. El método óptimo de alisamiento propuesto tiende a dar una estimación real de la superficie de las zonas de perturbaciones de depósitos de fosfato con una significativa reducción de ruido. Una característica particular del filtrado FTH es su habilidad para ajustarse a las discontinuidades abruptas en los datos. Los resultados muestran una supresión significativa del ruido y una muy buena recuperación de la señal de anomalías de resistividad. Esta técnica de alisado de mapas de datos de resistividad fue encontrada consistentemente útil y el mapa correspondiente pueden ser utilizados como herramientas auxiliares para la toma de decisiones bajo condiciones de campo.

\section{REFERENCIAS}

Bakkali, S. (2005). Analysis of Phosphate Deposit "Disturbances" using the HorizontalGradient Responses of Resistivity Data (Oulad Abdoun, Morocco). Earth Sciences Research Journal, Vol. 09, No. 02, pp. 123-131.

Bakkali, S. (2006). Optimización de Anomalias en un Depósito de Fosfatos de Marruecos mediante el Método del Sombreado. Stvdia Geologica Salmanticensia, Vol. 42, pp. 21-32.

Bakkali, S. (2007). Enhancement of Edges of Sidi Chennane Phosphate "Disturbances" using Sunshading Responses of Resistivity Data.
Russian Geology and Geophysics, Vol. 48, No. 09, pp. 775-781.

Bakkali, S. y L. Bahi (2006). Enhancement Edges of Sidi Chennane Phosphate Deposit "Disturbances" using Non-parametric Regression Analysis of Resistivity Data. Annals of the Faculty of Engineering Hunedoara, Tome IV, Fascicule 03, pp.135-140.

Bracewell, R.N. (1965). The Fourier Transform and Its Applications. pp. 381. McGraw-Hill Science/Engineering/Math. New York, USA.

Christensen, N.B. (1990). Optimized Fast Hankel Transform Filters. Geophysical Prospecting, Vol. 38, No. 05, pp. 545-548.

Johensen, H.K. y K. Sørensen (1979). Fast Hankel Transforms. Geophysical Prospecting, Vol. 27, No. 04, pp. 876-901.

Sørensen, K.I. y N.B. Christensen (1994). The Fields from a Finite Electrical Dipole-A New Computational Approach. Geophysics, Vol. 59, No. 06, pp. 864-880.

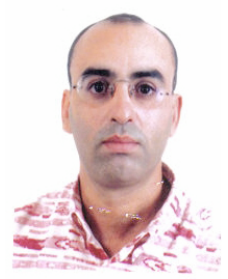

Saad Bakkali, es ingeniero en geofísica graduado por el Institut de Physique du Globe de Strasbourg, Université Louis Pasteur Strasbourg, Francia, donde también realizó una maestría en geofísica y geoquímica fundamental y aplicada. Profesor de geofísica aplicada y procesamiento de señales, Facultad de las Ciencias y Técnicas de Tánger, Marruecos. Su área de trabajo es procesamiento de señales aplicadas a datos geofísicos.

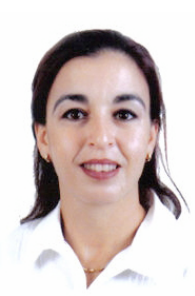

Mahacine Amrani, es
ingeniera en procesos
industriales graduada por Ecole
Mohammadia des Ingenieurs de
Rabat, Marruecos. Profesor de
procesos industriales y
procesamiento de señales, Facultad de las Ciencias y Técnicas de Tánger, Marruecos. Sus áreas de trabajo son procesamiento de señales y procesos industriales. 